\title{
Bilateral Dislocation of Mandibular Condyles following General Anesthesia-An Overlooked Problem: A Case Report
}

\author{
Mohd Toufeeq ${ }^{1}$ Murali Venkata Rama Mohan Kodali \\ Kavya Surapaneni ${ }^{1}$ \\ ${ }^{1}$ Department of Oral and Maxillofacial Surgery, Drs. Sudha and \\ Nageswara Rao, Siddhartha Institute of Dental Sciences, Krishna, \\ Andhra Pradesh, India \\ ${ }^{2}$ Department of Oral and Maxillofacial Surgery, College of Dentistry, \\ King Faisal University, Al Hofuf, Kingdom of Saudi Arabia \\ ${ }^{3}$ Department of Oral and Maxillofacial Surgery, All India Institute of \\ Medical Sciences, Bhubaneswar, Odisha, India
}

Srikanth Gunturu ${ }^{1}$ Kiran Kumar ${ }^{3}$

\begin{abstract}
Address for correspondence Mohd Toufeeq, MDS, Department of Oral and Maxillofacial Surgery, Drs. Sudha and Nageswara Rao, Siddhartha Institute of Dental Sciences (e-mail: mtoufeeq47@gmail.com).
\end{abstract}

Eur J Dent 2019;13:291-293
Abstract
Keywords
- dislocation
- condylectomy
- temporal fossa

Dislocation of mandibular condyles can occur following excessive mouth opening or traumatic injury to the temporomandibular joint. It can also occur during general anesthesia that at times may go un-noticed in the modern-day theater setup. Here, we describe a case of bilateral dislocation of mandibular condyle following orotracheal intubation for general anesthesia. Right condyle was dislocated into temporal fossa.

\section{Introduction}

Temporomandibular joint (TMJ) dislocation is the clinical condition where the head of the condyle is displaced out of the glenoid fossa. ${ }^{1}$ It represents $\sim 3 \%$ of all dislocated joints in the body. ${ }^{2}$ Patients usually present with pain in the preauricular region, difficulty in closing the mouth, anterior open bite, and deviated chin, causing severe pain and discomfort to the patient.

Usually, it occurs when the TMJ is subjected to excessive movement beyond its normal range ${ }^{3}$ or following any traumatic injury to the TMJ. ${ }^{4}$ Similarly, mandibular condyle tends to get dislocated by excessive maneuvering during tracheal intubation under general anesthesia..$^{5-7}$ It can occur at any time during laryngoscopy while intubation or extubation. ${ }^{6}$ Here, we report a case of dislocation of TMJ following tracheal intubation in a 60-year-old woman that was overlooked for a prolonged period necessitating the resection of the condyle.

Anesthesiologists, surgical team, and other health professionals should be aware regarding the prevention, early diagnosis, and manual reduction in condyle into the glenoid fossa at the earliest to preclude an aggressive treatment as mentioned in the present case.

\section{Case Presentation}

A 60-year-old woman reported to our department with difficulty in closing the mouth for the last 4 weeks. History of present illness reveals difficulty in closing the mouth, chewing, and speaking following hysterectomy under general anesthesia 4 weeks ago.

The patient was treated with analgesics and muscle relaxants during the postoperative period following hysterectomy. Later, when the symptoms were not subsided for an extended period of time, the patient was referred to the dentist for a complete examination. The patient was suspected to have dislocations of the mandibular condyles and the closed manual reduction was attempted several times under local anesthesia by the dentist that was unsuccessful.

Clinical examination revealed hollowness in the bilateral preauricular region with restricted lower jaw movement and inability to close or open mouth. A three-dimensional computed tomography showed dislocated condyle in the anterior direction on the left side and into the temporal fossa on the right side ( - Fig. 1A, B).

Under general anesthesia, the manual reduction was performed. Left condyle was reduced into the glenoid fossa 

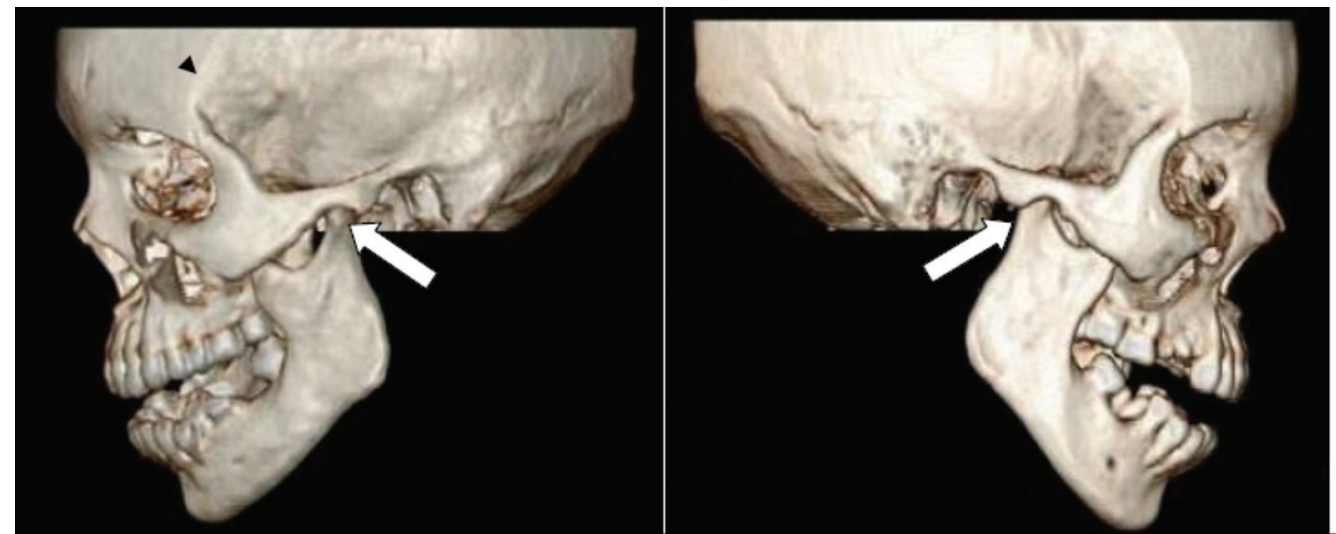

Fig. 1 Three-dimensional CT images depicting dislocation of condyles in the anterior direction on left side and in the temporal fossa on the right side (white arrows). CT, computed tomography.

manually but not on the right side. So, the TMJ on the right side was opened through the preauricular incision. Dislocation of right mandibular condyle into the temporal fossa was noticed on exposing the joint; manual reduction could not be achieved as it was being obstructed by the zygomatic arch. Hence, condylectomy was done on the right side (-Fig. 2).

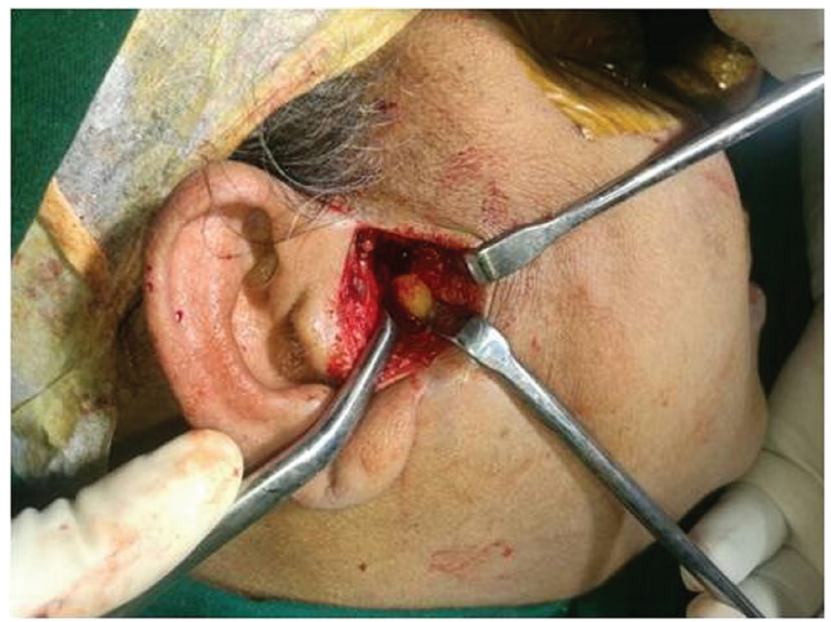

Fig. 2 Intraoperative image showing condylectomy on right side.
Immobilization of the mandible using eyelet wiring was done for 4 weeks post condylectomy and then physiotherapy was initiated. A postoperative panoramic radiograph was taken to assess the TMJ ( - Fig. $\mathbf{3}$ ).

Patient was followed up for a period of 6 months. There was no difficulty in closing and opening the mouth during the follow-up period. Slight deviation of mandible on the right side was observed.

\section{Discussion}

Dislocation of mandibular condyles following general anesthesia has been mentioned in the literature. ${ }^{5-7}$ It is often unnoticed unless patient complains of pain and swelling during the postoperative period. Difficulty in opening and closing the mouth for a prolonged period of time causes severe discomfort and embarrassment to the patient.

Unawareness of the anesthesiologists or surgical team with the dislocation of TMJ seems to be contributing factor for delayed diagnosis and its treatment. ${ }^{6}$

Prabhakar and Sigla ${ }^{8}$ reported a case of bilateral dislocation of condyles into the temporal fossa following an injury to the chin region. The presence of rounded mandibular condyle and elasticity of zygomatic arch are considered to be the

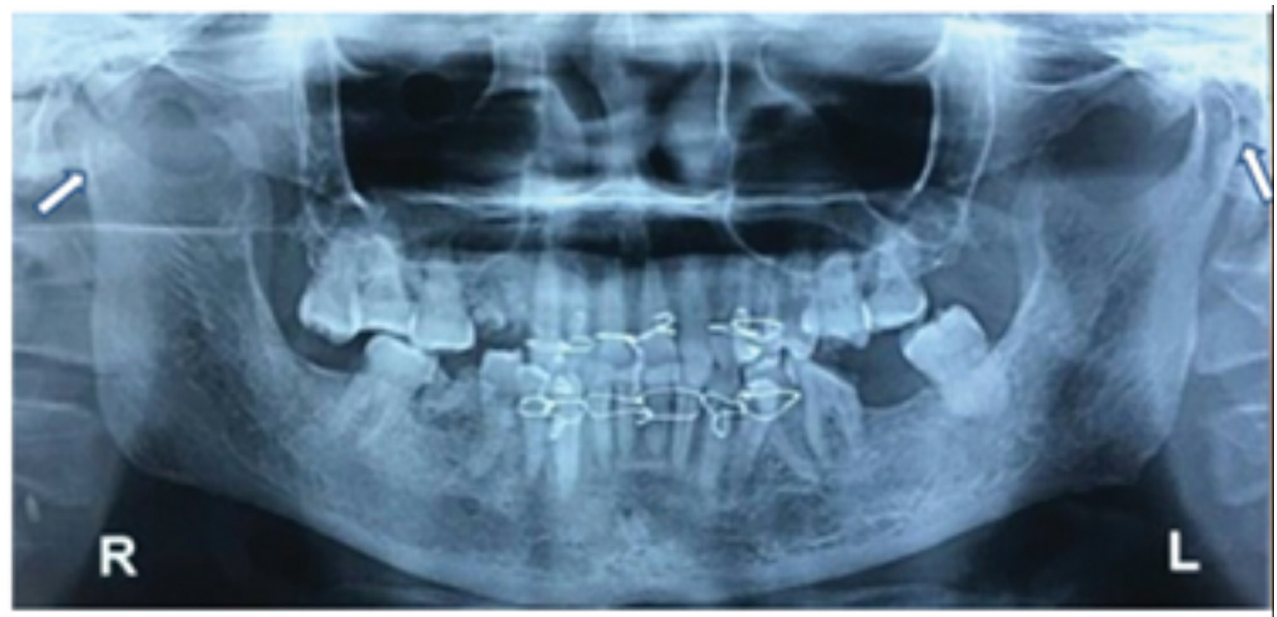

Fig. 3 Postoperative panoramic radiograph showing condylectomy on right side and condyle in the glenoid fossa on left side (white arrows). 
contributing factors for dislocation of mandibular condyle into the temporal fossa. ${ }^{9}$

The simple manual reduction is considered to be the first treatment option for any type of dislocation. Any delay in the reduction may induce fibrosis of the soft tissues around the joint that may further make reduction difficult and necessitates condylectomy, ${ }^{9}$ which in turn reduces ramus height and deviation of the mandible toward affected side.

In the present case, manual reduction was planned under general anesthesia as the patient was apprehensive due to previous repeated attempts toward repositioning of condyles under local anesthesia. Left condyle could be reduced into glenoid fossa but not on right side. Difficulty in reducing condyle on the right side may be due to the presence of fibrous tissue and obstruction of condyle by zygomatic arch.

\section{Conclusion}

There is a possibility of mandibular condyles getting dislocated during tracheal intubation under general anesthesia. Anesthesiologists, surgical team, and other health professionals should be aware of this iatrogenic complication such that early intervention can be initiated.

\section{Conflict of Interest}

None declared.

\section{References}

1 Baldwin AJ. Superior dislocation of the intact mandibular condyle into the middle cranial fossa. J Oral Maxillofac Surg 1990;48(6):623-628

2 Shorey CW, Campbell JH. Dislocation of the temporomandibular joint. Oral Surg Oral Med Oral Pathol Oral Radiol Endod 2000;89(6):662-668

3 Thangarajah T, McCulloch N, Thangarajah S, Stocker J. Bilateral temporomandibular joint dislocation in a 29-year-old man: a case report. J Med Case Reports 2010;4:263

4 Allen FJ, Young $\mathrm{AH}$. Lateral displacement of the intact mandibular condyle. A report of five cases. Br J Oral Surg 1969;7(1):24-30

5 Han I, Kim TK, Yoo J-H, Park JH, Chung EY. Dislocation of the temporomandibular joint following general anesthesia. Korean J Anesthesiol 2014;67(Suppl):S113-S114

6 Pillai S, Konia MR. Unrecognized bilateral temporomandibular joint dislocation after general anesthesia with a delay in diagnosis and management: a case report. J Med Case Reports 2013;7:243

7 Wang LK, Lin MC, Yeh FC, Chen YH. Temporomandibular joint dislocation during orotracheal extubation. Acta Anaesthesiol Taiwan 2009;47(4):200-203

8 Prabhakar V, Singla S. Bilateral anterosuperior dislocation of intact mandibular condyles in the temporal fossa. Int J Oral Maxillofac Surg 2011;40(6):640-643

9 Bu SS, Jin SL, Yin L. Superolateral dislocation of the intact mandibular condyle into the temporal fossa: review of the literature and report of a case. Oral Surg Oral Med Oral Pathol Oral Radiol Endod 2007;103(2):185-189 